\title{
Cross-cultural adaptation and validation of the Liebowitz Social Anxiety Scale (LSAS-SR) Sinhala Version
}

\author{
Aruni Hapangama ${ }^{1}$, Lalith A Kuruppuarachchi ${ }^{1}$, Ransirini de Silva ${ }^{2}$, Ananda R Wickremasinghe ${ }^{3}$, \\ Arun Ravindran ${ }^{4}$, Shehan S Williams ${ }^{1}$
}

\section{Background}

Social phobia or social anxiety disorder is characterized by a fear of scrutiny and avoidance of a variety of social and performance situations $[1,2]$. In Western societies, it is considered to be among the most common anxiety disorders in adults with a lifetime prevalence of $7-13 \%[3,4]$ and is reported as the third most common psychiatric disorder after major depression and alcohol dependence syndrome [3]. Its prevalence among university students is reported to be between $8-26 \%$ [5-7]. Untreated, social phobia is reported to be chronic and unremitting, and also reported to contribute to academic, occupational and relationship problems $[4,6,8]$. Despite the relatively high prevalence and impairments associated with social phobia, most people with the disorder do not seek treatment and, even if they do, they are reported to be unlikely to receive empiricallysupported therapies [9].

Several instruments have been developed to screen or measure the severity of social phobia, including the Liebowitz Social Anxiety Scale - clinician assisted version (LSAS-CA) [10], MINI-Social phobia Inventory [11] and Social Phobia Inventory [12].

The LSAS-CA has been reported as one of the most frequently used measures of social phobia in adults as well as one of the best psychometrically validated scales for this disorder [13]. Despite good validity and reliability, the LSAS-CA is reported to be relatively costly because it can only be administered by a skilled clinician [14]. This had led to the development of the self-reported version of the Liebowitz Social Anxiety Scale (LSAS-SR) and its psychometric qualities are reported to be as satisfactory as the clinician-administrated version [15]. The LSAS-SR has been shown to have good internal consistency (Chronbach's $\alpha=0.81-0.96$ ), and good convergent and discriminant validity for the total score as well as for the two subscales of fear and avoidance [15]. LSAS-SR has been adapted and validated into French [16], Spanish [17] and Turkish [18].

Data regarding the prevalence and impact of social phobia among Sri Lankans are scarce. It is therefore important to identify and validate an instrument for screening social phobia in a native language such as Sinhala in Sri Lanka. Western-developed scales may not be suitable for populations with different socio-cultural and linguistic constructs and hence need to be adapted and validated for different ethnic groups. Given the high prevalence of social phobia reported among university students in other countries [5, 6, 7] and the significant negative impact of untreated social phobia among this subgroup, we aimed to culturally adapt and validate the LSAS-SR in Sinhala using a group of Sri Lankan university students.

\section{Methodology}

The study was conducted among students of the Universities of Kelaniya and Moratuwa in Sri Lanka following approval by the Ethics Review Committee of the Faculty of Medicine, University of Kelaniya (P/60/05/2015). Participants who were willing to provide written informed consent were recruited to the study.

The LSAS-SR consists of two subscales of 24 items on fear and avoidance in social and performance situations scored on a four point Likert-type scale and evaluates symptoms experienced in the week prior to scale administration.

The LSAS-SR was translated and adapted into Sinhala according to standard technical recommendations. The instrument was first translated from its original English

Ceylon Medical Journal 2020; 65: 28-31

DOI: http://doi.org/10.4038/cmj.v65i1-2.9134

${ }^{1}$ Department of Psychiatry, Faculty of Medicine, University of Kelaniya, ${ }^{2}$ University of Kelaniya, ${ }^{3}$ Department of Public Health, Faculty of Medicine, University of Kelaniya, ${ }^{4}$ Director of Global Mental Health Affairs and the Office of Fellowship Training, Department of Psychiatry, University of Toronto, Canada.

Correspondence: AH, e-mail: <ahapangama@yahoo.co.uk>. Received 27 January 2020 and revised version 27 June 2020 accepted 02 July 2020.

This is an open-access article distributed under the terms of the Creative Commons Attribution License, which permits unrestricted use, distribution, and reproduction in any medium, provided the original author and source are credited. 
version into Sinhala by two consultant psychiatrists with good knowledge of both languages. This version was then independently back translated by another two consultant psychiatrists, and was presented to the authors of the original scale for comparison. The versions were compared and discussed by two bilingual consultants with experience in the rating of anxiety disorders and in psychiatric evaluation scales; they performed the verification of semantic equivalence and, after reaching a consensus, proposed the translated version of the scale. Face validity and cultural acceptability of the translation was established using the Delphi technique among a panel of experts. The semantics of the words and the general structure of the final Sinhala version of the instrument were in close agreement with that of the original English version. The translated version was pre-tested for alternate form reliability among 54 participants from the study population. Respondents who completed the scale were asked via a verbal open-ended question to elaborate what they thought about each item of the scale and what their corresponding response meant. This was done to ensure that the translated items retained the same meaning as the original items and that there was no confusion regarding the items of the translated questionnaire among respondents. The respondents did not have major queries regarding the items or the corresponding response. The final version was presented to Prof Michael R. Liebowitz, (author of the original scale) for review.

\section{Sample size}

In order to estimate a sensitivity and specificity of $90 \%$, with an alpha error of 0.05 and confidence interval ranging from $82.5 \%$ to $97.5 \%, 62$ persons with and 62 persons without social phobia were required. As the prevalence of social phobia has been reported to be around $10 \%, 620$ persons needed to be screened. Those identified with social phobia, depression or any mental health issue were offered informtion and referred for treatment irrespective of study participation status.

A different group of participants among consenting students were administered the newly-translated Sinhala LSAS-SR. A consultant psychiatrist blind to the responses on the LSAS-SR conducted an independent interview of the participants using DSM-IV [1] diagnostic criteria which were considered as the gold standard to arrive at a diagnosis of social phobia.

To assess the test-retest reliability, participants with minimal changes on the Clinical Global Impression (CGI) scale after two weeks were administered the Sinhala translation of the LSAS-SR by an investigator blind to the initial results of the LSAS-SR Sinhala version.

Statistical analysis - The data were manually coded and stored in a database. SPSS version 17.0 was used for data analysis. DSM-IV criteria assessed by a consultant psychiatrist was used as the gold standard to assess criterion validity. Cronbach's alpha was calculated to assess internal consistency between the subscales (avoidance and fear) and the total scale to assess internal consistency of the whole tool. Pearson's correlation coeffient was used to assess test-retest reliability.

\section{Results}

A total of 661 students (male 271, female 390) from Medicine $(n=318)$ and Speech and Hearing Sciences ( $n=186)$ streams of the Faculty of Medicine, University of Kelaniya, and engineering $(n=157)$ students of the University of Moratuwa participated (Table 1).

Table 1. Characteristics of the sample

\begin{tabular}{|c|c|c|c|c|}
\hline Variable & $\begin{array}{l}\text { Number (\% total } \\
\text { sample size) }\end{array}$ & $\begin{array}{c}\text { Number without social } \\
\text { phobia (\% sample) as } \\
\text { as per DSM IV }\end{array}$ & $\begin{array}{c}\text { Number with social } \\
\text { phobia (\% sample) } \\
\text { as per DSM IV }\end{array}$ & $\begin{array}{c}\text { Chi square statistic } \\
(p \text {-value })^{*}\end{array}$ \\
\hline \multicolumn{5}{|l|}{ Gender } \\
\hline Male & 271 (40.99) & $248(37.5)$ & $23(8.48)$ & $1.31(0.25)$ \\
\hline Female & $390(59.00)$ & 346 (52.3) & $44(11.28)$ & \\
\hline \multicolumn{5}{|l|}{ Age group } \\
\hline 19-24 years & 492 (74.43) & 437 (66.1) & $55(8.3)$ & $2.297(0.130)$ \\
\hline $25-30$ years & $169(25.57)$ & 157 (23.8) & $12(1.8)$ & \\
\hline \multicolumn{5}{|l|}{ Course of Study } \\
\hline Medicine & $318(48.11)$ & $279(42.2)$ & 39 (5.9) & $11.6(0.003)$ \\
\hline Engineering & 157 (23.75) & 136 (20.6) & $21(3.2)$ & \\
\hline \multicolumn{5}{|l|}{ Speech and } \\
\hline Hearing Sciences & $186(28.14)$ & $179(27.1)$ & $7(1.1)$ & \\
\hline \multicolumn{5}{|l|}{ Year of Study } \\
\hline First year & 327 (49.47) & $299(45.2)$ & $28(4.2)$ & $4.3(0.37)$ \\
\hline Second year & 154 (23.29) & $135(20.4)$ & 19 (2.9) & \\
\hline Third year & $73(11.04)$ & $64(9.7)$ & $9(1.4)$ & \\
\hline Fourth year & $61(9.23)$ & $57(8.6)$ & $4(0.6)$ & \\
\hline Fifth year & $46(6.96)$ & 39 (5.9) & $7(1.1)$ & \\
\hline
\end{tabular}

*denotes $\mathrm{p}<=0.05$ 
As per the DSM-IV criteria, the prevalence of social phobia was $10.14 \%$ among the whole population with a prevalence of $11.28 \%$ among female students and $8.48 \%$ among male students. The area under the receiver operating characteristics curve was $87.8 \%$ (Figure 1 ).

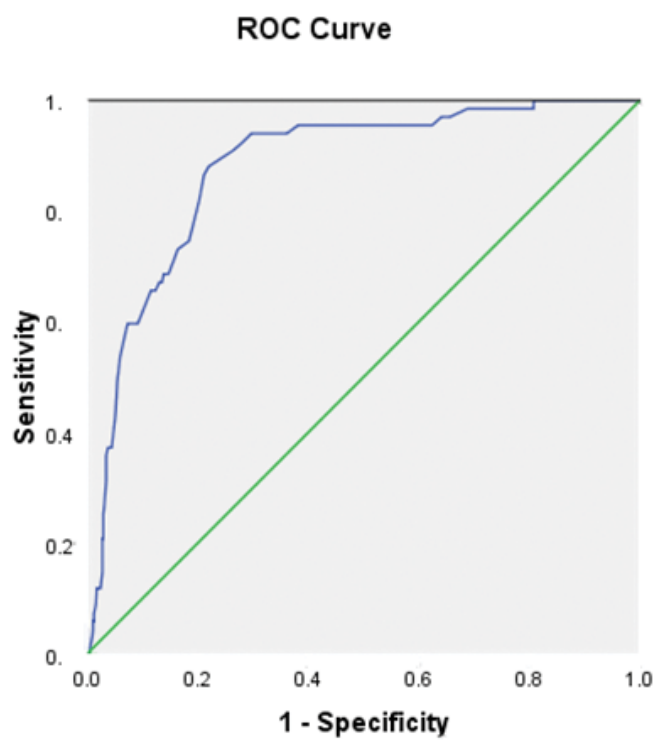

Figure 1. Receiver Operating Characteristics curve for the LSAS-SR.

Table 2 gives the sensitivities and specificities for different cutoff values. A total score of 39 provided best balance between sensitivity (82.1\%) and specificity (79.6\%). The false positive rate was $21.2 \%$ and the Positive Predictive Value was $46.0 \%$.

Table 2. Sensitivities and specificities for different cutoff points based on the receiver operating characteristics curve

\begin{tabular}{ccc}
\hline Cutoff value & Sensitivity & Specificity \\
\hline 30.50 & .940 & 0.668 \\
31.50 & .940 & 0.680 \\
32.50 & .940 & 0.694 \\
33.50 & .940 & 0.702 \\
34.50 & .925 & 0.717 \\
35.50 & .910 & 0.734 \\
36.50 & .881 & 0.779 \\
38.00 & .866 & 0.788 \\
39.50 & .821 & 0.796 \\
40.50 & .746 & 0.815 \\
41.50 & .731 & 0.835 \\
42.50 & .687 & 0.852 \\
43.50 & .687 & 0.860 \\
44.50 & .672 & 0.864 \\
\hline
\end{tabular}

The Cronbach's alpha values for the avoidance subscale, fear subscale and the total score were 0.999, 0.998 and 0.994 , respectively.

Pearson's correlation coefficient for test-retest reliability was $0.998,0.998$ and 0.994 for fear subscale, avoidance subscale and total sore, respectively.

The prevalence of social phobia among the study population was $13.3 \%$ based on the LSAS-SR Sinhala version using a cut of score of 39.

\section{Discussion}

The Sinhala version of LSAS-SR had good test-retest reliability and adequate internal consistency for the fear and avoidance subscales and the total score.

Our results are comparable to that of Baker et al [15] who reported overall good results of test-retest reliability and internal consistency. In addition, the results of the validation of the Sinhala version LSAS is comparable to the French [16], Spanish [17] and the Turkish [18] versions which also showed good cross-cultural consistency.

The cut off score of 39 for the LSAS-SR Sinhala version gave the optimal sensitivity and specificity for social phobia. The original LSAS-SR reports a cut off score above 50 as moderate social phobia whereas the study by Mennin et al [19] reported that a cut off score of 30 is sufficient to detect social phobia in clinical settings. The original version categorizes the total score as moderate, marked, severe and very severe if the total score is in the ranges of 50-65, 65-80, 80-95 and > 95, respectively. However, it does not have a category for milder symptoms.

$13.3 \%$ of the students in our study group had social phobia according to the LSAS-SR and this figure is relatively higher than figures reported from university students from some Western countries [5]. However studies done among university students in India and Saudi Arabia have shown much higher prevalences (25\% [6] and $25.8 \%$ [7], respectively). We postulate that the higher prevalence of social phobia among our study population when compared with the West may stem from the allocentric characteristics of the Asian culture, where individuals fear offending or distressing others [20]. We also postulate that the higher prevalence of social phobia observed in our study population may also be explained by the following phenomenon. Most of the students in Sri Lanka have their primary and secondary education in their mother tongue (either Sinhala or Tamil). However, once they enter university, students who follow university courses in Sri Lanka especially in medicine, speech and hearing sciences and engineering are expected to conduct their studies exclusively in the English medium. Lack of mastery of the English language in some students within this population may have led them to experience social or performance anxiety when having to communicate in English in front of lecturers and or peers. Furthermore, Sri Lankan parenting styles and other socialization 
experiences including abuse or harassment by senior students and socioeconomic conditions may also have contributed to our findings.

\section{Limitations}

The LSAS-SR Sinhala version was validated only among university students who could read and write Sinhala. The literacy state of the person may have an impact on the score and the score may vary if conducted among the general public.

\section{Conclusion}

The cut off score of 39 had the best sensitivity and specificity for the LSAS-SR Sinhala version. It had good internal consistency and test-retest reliability. Translating LSAS- SR into Tamil and further studies will enable the assessment of the prevalence of social phobia in the Sri Lankan population as a whole and investigate the cultural and environmental factors associated with social phobia in this particular population of university students.

\section{Acknowledgements}

This study was supported by a grant from the National Research Council of Sri Lanka (grant number 13-115) and a University research grant from the University of Kelaniya, Sri Lanka (grant number 03/04/14/01/2015).

\section{Conflicts of interest}

There are no conflicts of interest.

\section{References}

1. American Psychiatric Association. Diagnostic and statistical manual for mental disorders (DSM). 4th edn. Washington, DC: American Psychiatric Press, Inc, 1994.

2. WHO. International statistical classification of diseases and related health problems, 10th Revision. World Health Organization, 1992.

3. Kessler RC, Chiu WT, Demler O, Walters EE. Prevalence, severity, and comorbidity of 12-month DSM-IV disorders in the National Comorbidity Survey Replication. Arch Gen Psychiatry 2005; 62: 617-27

4. Fehm L, Pelissolo A, Furmark T, et al. Size and burden of social phobia in Europe. Eur Neuropsychopharmacol 2005; 15: 453-62.

5. Tillfors M, Furmark T. Social phobia in Swedish university students: prevalence, subgroups and avoidant behavior. Soc Psychiatry Psychiatr Epidemiol 2007; 42(1): 79-86.
6. Mascarenhas JJ Krishna A, Pinto D. Social Phobia (Social Anxiety Disorder) in Medical and Paramedical First Year Undergraduates. Gal Int J Health Sci Res 2019; 4(2); 120-6.

7. Hakami RM, Mahfouz MS, Adawi AM, et al. Social anxiety disorder and its impact in undergraduate students at Jazan University, Saudi Arabia. MentIlln 2018; 9(2): 7274.

8. Timothy JB, Atezaz S. Social anxiety disorder: a common unrecognized mental disorder. Am Fam Physician 1999; 60(8): 2311-2319

9. Olfson M, Guardino M, Struening E, et al. Barriers to treatment of social anxiety. Am J Psychiatry 2000; 157: 521-7.

10. Liebowitz MR. Social phobia. Mod Probl Pharmacopsychiatry 1987; 22: 141-73.

11. Connor KM, Kobak KA, Churchill LE, et al. A brief screening assessment for generalized social anxiety disorder. 2001; 14(2): 137-40.

12. Connor KM, Davidson JRT, Churchill LE, et al. Psychometric properties of the Social Phobia Inventory. Br J Psychiatry 2000; 176: 379-86.

13. Heimberg RG, Horner KJ, Juster HR, et al. Psychometric properties of the Liebowitz Social Anxiety Scale. Psychol Med 1999; 29: 199-212.

14. Rytwinski NK, Fresco DM, Heimberg RG, et al. Screening for social anxiety disorder with the self-report version of the Liebowitz Social Anxiety Scale. Depress Anxiety 2009; 26(1): 34-8.

15. Baker SL, Heinrichs N, Kim HJ, Hofmann SG. The Liebowitz social anxiety scale as a self-report instrument: a preliminary psychometric analysis. Behav Res Ther 2002; 40: 701-15.

16. Yao S, Note I, Fanget F, Albuisson E, et al. L 'anxiétésociale chez les phobiquessociaux: Validation de l'échelled' anxiétésociale de Liebowitz (version française). L’encéphale. 1999; 25: 429-35.

17. Bobes J, Badia X, Luque A, Garcia M, Gonzales MP, DalRé R. Validation of the Spanish version of the Liebowitz social anxiety scale, social anxiety and destress scale and Sheehan disability inventory for the evaluation of social phobia. Med Clin 1999; 112: 530-8.

18. Soykan Ç, Özgüven HD, Gençöz T Liebowitz Social Anxiety Scale: the Turkish Version. Psychol Rep 2002; 93: 105910-69.

19. Mennin DS, Fresco DM, Heimberg RG, Schneier FR, Davies SO, Liebowitz MR. Screening for social anxiety disorder in the clinical setting: using the Liebowitz Social Anxiety Scale. J Anxiety Disord. 2002; 16(6): 661-73.

20. Choy Y, Schneier FR, Heimberg RG, et al. Features of the offensive subtype of Taijin-Kyofu-Sho in US and Korean patients with DSM-IV social anxiety disorder. Depress Anxiety 2008; 25: 230-40. 